\title{
Structural plasticity, cortical memory, and the spacing effect Andreas Knoblauch
}

\author{
Address: Honda Research Institute Europe, Offenbach, Germany \\ Email: Andreas Knoblauch - andreas.knoblauch@honda-ri.de
}

from Eighteenth Annual Computational Neuroscience Meeting: CNS*2009

Berlin, Germany. 18-23 July 2009

Published: 13 July 2009

BMC Neuroscience 2009, I0(Suppl I):O16 doi:I0.1 I86/I47|-2202-I0-SI-OI6

This abstract is available from: http://www.biomedcentral.com/I47I-2202/I0/SI/OI6

(c) 2009 Knoblauch; licensee BioMed Central Ltd.

The neurophysiological basis of learning and memory is commonly attributed to the modification of synaptic strengths in neuronal networks. Recent experiments suggest also a major role of structural plasticity, including elimination and regeneration of synapses, growth and retraction of dendritic spines, and remodeling of axons and dendrites [1]. Here, I develop a simple model of structural plasticity and synaptic consolidation in neural networks and apply it to Willshaw-type network models of distributed associative memory [2]. The model assumes synapses with discrete weight states. Synapses with low weights have a high probability of being erased and replaced by novel synapses at other locations. In contrast, synapses with large weights are consolidated and cannot be erased. Analysis and numerical simulations reveal that this model can explain various cognitive phenomena much better than alternative network models employing synaptic plasticity only.

In previous work, I have shown that networks with low anatomical connectivity employing structural plasticity in coordination with stimulus repetition (e.g., by hippocampal memory replay) can store much more information per synapse by "emulating" high effective memory connectivity close to potential network connectivity [2]. In this work, I present additional simulations and analyses suggesting that networks employing structural plasticity suffer to a much lesser degree from catastrophic forgetting than models without structural plasticity [3] if the number of consolidated synapses remains sufficiently low. The reason for this effect is that early memories get stored with a higher effective connectivity than recent memories. Therefore the ability to learn new items fades gradually without affecting remote memories. The same effect may explain Ribot gradients in retrograde amnesia much better than previous models relying on gradients in replay time [4].

Another salient feature of memory is the spacing effect [5] where learning new items is more effective if rehearsal is spread out over time compared to the case when rehearsal is done in a single time block. For example, rehearsing a list of vocabulary two times for ten minutes each is more effective than doing a single rehearsal for twenty minutes. The spacing effect is a remarkably robust phenomenon and can be found in many explicit and implicit memory tasks in humans and many animals being effective over many time scales from single days to months. This suggests that there should be a very general learning mechanism underlying the spacing effect. However, current explanations rather suggest very specific mechanisms referring to detailed implementations of memory systems including attention, novelty, and context processing. While these explanations may well account for some detailed characteristics of the spacing effect in some specific memory tasks, I suggest that the common underlying mechanism at the cellular level is structural plasticity in sparsely connected neural networks. This is supported by simulation experiments and analyses showing that simple models of structural plasticity and consolidation robustly reproduce the spacing effect. The interpretation is that ongoing structural plasticity can reorganize the network during the long time intervals between two rehearsal periods by growing a lot of new synapses at potentially useful locations. Therefore, subsequent training can strongly increase effective memory connectivity and thus reduce 
retrieval noise. In contrast, single block rehearsal can increase effective memory connectivity only slightly above anatomical connectivity. This leads to the hypothesis that the spacing effect is as robust and general as observed in various experimental paradigms because structural plasticity is a ubiquitous feature of many neural networks in the human and animal brain. The spacing effect occurs over many time scales because also structural plasticity works over many different time scales corresponding to elimination and regeneration of synapses, spines, axons, and dendrites, ranging from minutes to months or even years.

\section{References}

I. Chklovskii DB, Mel BW, Svoboda K: Cortical rewiring and information storage. Nature 2004, 43 I:782-788.

2. Knoblauch $A$ : On compressing the memory structures of binary neural associative networks. Honda Research Institute Europe Technical Report HRI-EU 06-02 2006.

3. Robins A, McCallum S: Catastrophic forgetting and the pseudorehearsal solution in Hopfield type networks. Connection Science 1998, 7:121-135.

4. Meeter M, Murre JMJ: TraceLink: A model of consolidation and amnesia. Cognitive Neuropsychology 2005, 22:559-587.

5. Crowder RG: Principles of learning and memory Oxford: Lawrence Erlbaum; 1976.
tral.com/1471-2202/10/S1/016

Publish with Biomed Central and every scientist can read your work free of charge

"BioMed Central will be the most significant development for disseminating the results of biomedical research in our lifetime. "

Sir Paul Nurse, Cancer Research UK

Your research papers will be:

- available free of charge to the entire biomedical community

- peer reviewed and published immediately upon acceptance

- cited in PubMed and archived on PubMed Central

- yours - you keep the copyright

Submit your manuscript here:

http://www.biomedcentral.com/info/publishing_adv.asp 